\title{
SURVEY OF HIGHER EDUCATION INSTITUTIONS INVOLVEMENT IN THE REGIONS OF GEORGIA IN THE CONTEXT OF GRADUATE EMPLOYMENT
}

\author{
NATELA TSIKLASHVILI \\ Doctor of Economics, Professor \\ Batumi Shota Rustaveli State University, Georgia \\ natia.tsiklashvili@bsu.edu.ge
}

\section{TAMARI POLADASHVILI}

PhD Student

Batumi Shota Rustaveli State University, Georgia

tamari.poladashvili@bsu.edu.ge

Abstract. Reforms in the field of education have been going on for almost 30 years since Georgia gained its independence. During the period, numbers of ministers and heads of educational institutions were replaced. Unfortunately, the subject of graduate employment remains an unresolved issue. Higher education institutions (HEIs) are crucial in training qualified graduates and employing them according their profession.

The given paper presents the qualitative analysis of Higher Education Institutions involvement in the context of graduate employment. The study involves all accredited universities and teaching universities located in the regions of Georgia by January 2020, which had at least first-generation of graduates. This approach determines the reliability and validity of the study. Hence, the research encompasses 4 universities and 6 teaching universities located in the regions of Georgia.

The aim of the research is to evaluating the current conditions of HEls in relation to labor market stakeholders, determine the problems and challenges in regards of graduates' employment by profession. Analyze the functions of career services and outline the aspects that create horizontal and vertical unemployment of graduates.

The presented article is one of the first academic attempt that examines region based Higher Educational Institutions by qualitative research method, using interview technique. Therefore, its scientific and practical significance is important in terms of the developing regional and then national higher educational environment.

KEYWORDS: HIGHER EDUCATION; EMPLOYMENT; GRADUATE COMPETITIVENESS; HIGHER EDUCATION INSTITUTIONS LOCATED IN THE REGIONS OF GEORGIA.

For citation: Tsiklashvili, N., Poladashvili, T. (2021). Survey of Higher Education Institutions Involvement in the Regions of Georgia in the Context of Graduate Employment. Globalization and Business. 12, 37-44. (In Georgian). https://doi. org/10.35945/gb.2021.12.004 


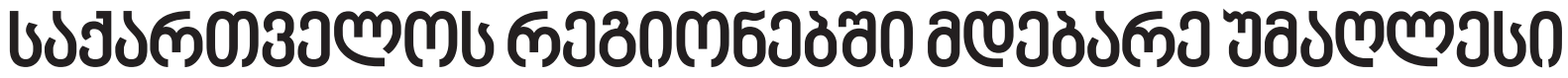

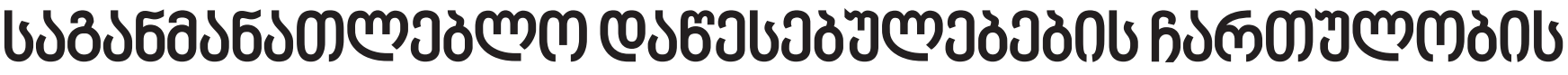

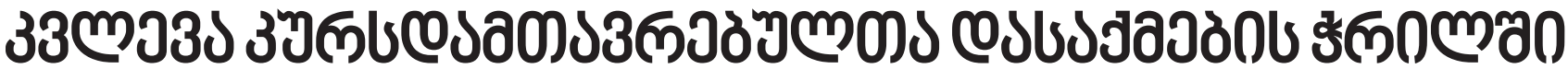

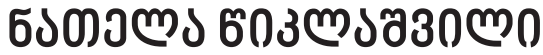

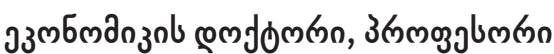

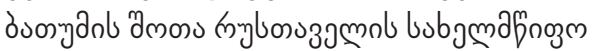

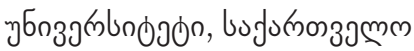

natia.tsiklashvili@bsu.edu.ge

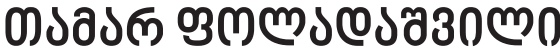

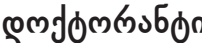

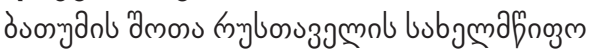

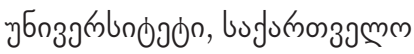

tamari.poladashvili@bsu.edu.ge

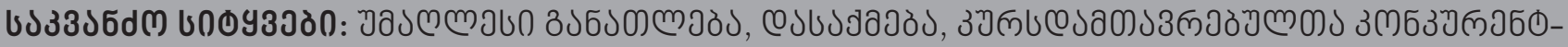

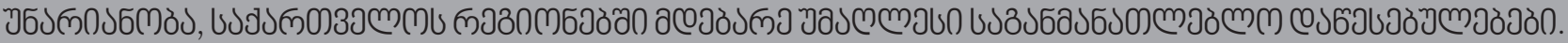

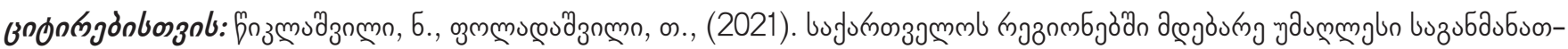

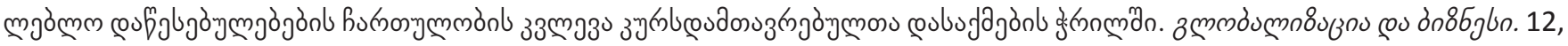
37-44. https://doi.org/10.35945/gb.2021.12.004

\section{ఇอ৮১З১ल0}

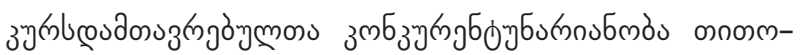

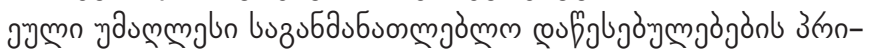

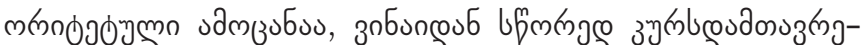

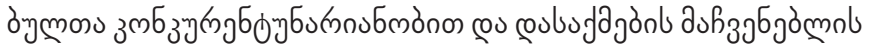

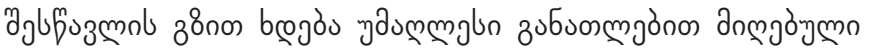

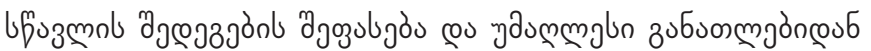

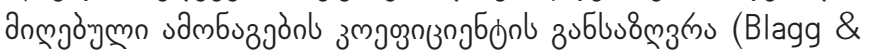
Blom, 2018; Hailemariam , 2018; Amashukeli, et al., 2017; Mincer, 1974).

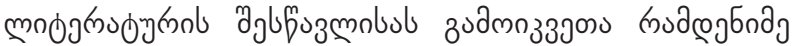

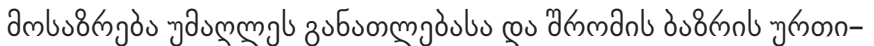

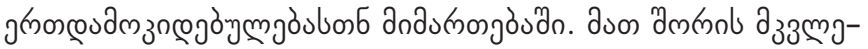
3

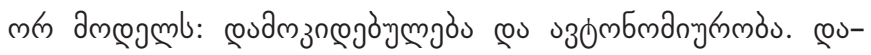

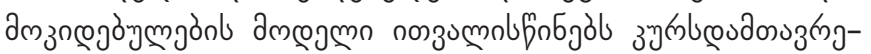

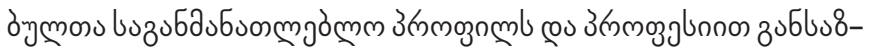

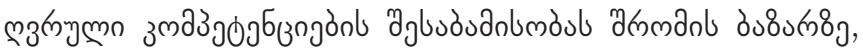

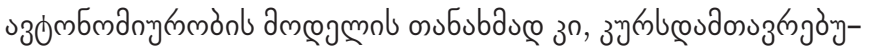

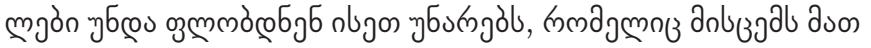

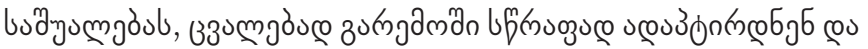

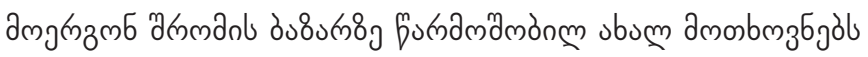
("successive and flexible adjustments").

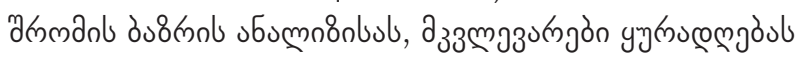

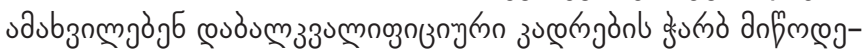

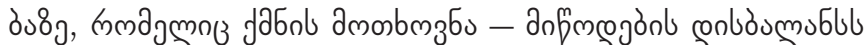
(Bregvadze, 2013; Amashukeli \& Lezhava, 2015; Kakulia,et

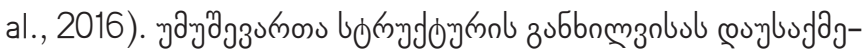

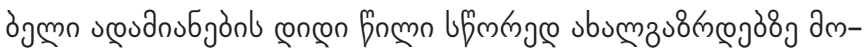
conl (Ministry of Economy and Sustainable Development

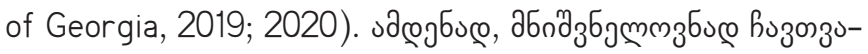

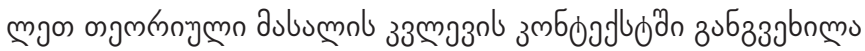

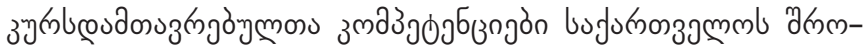
anl bu8mol amonbmз

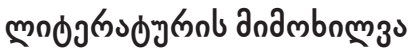

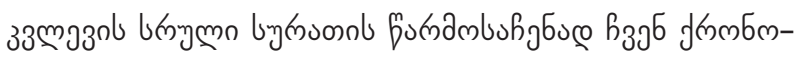

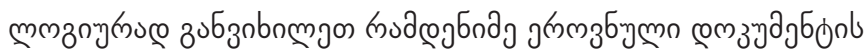
sढzumono:

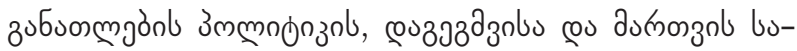

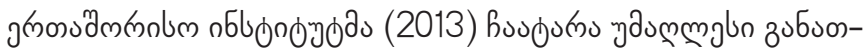

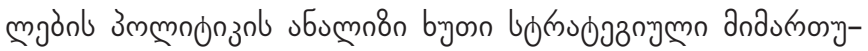




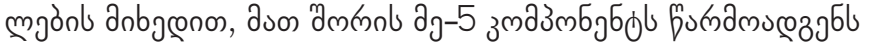

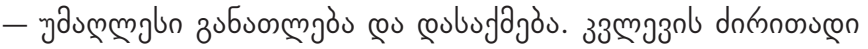

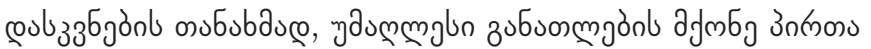

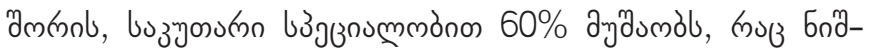

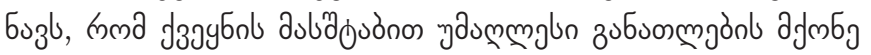

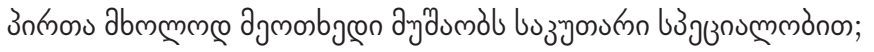

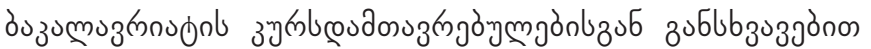

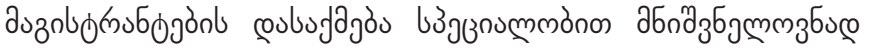

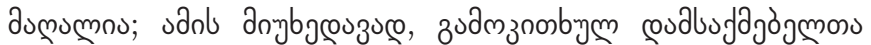

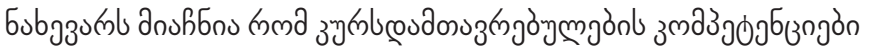

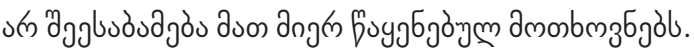

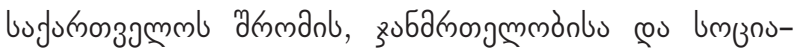

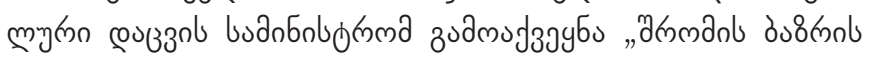

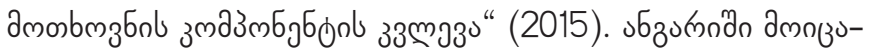

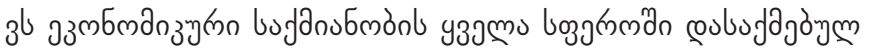

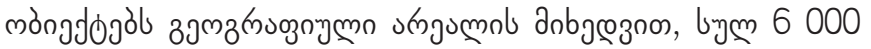

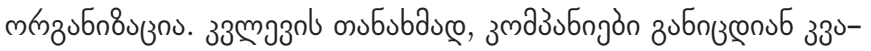

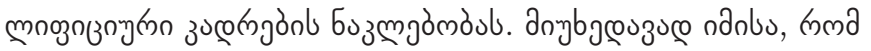

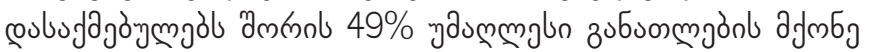

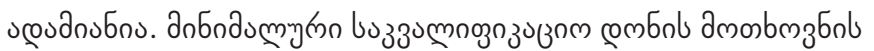

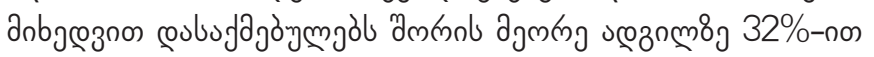

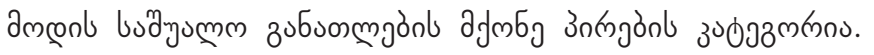

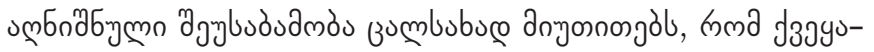

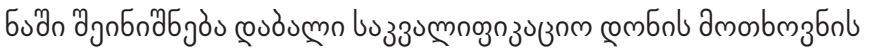

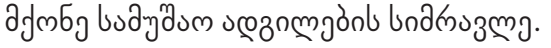

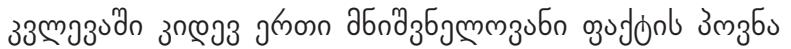

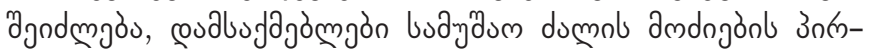

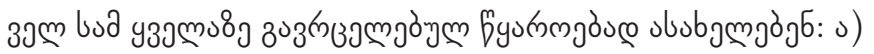

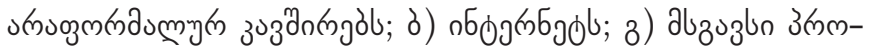

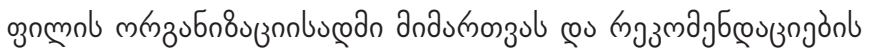

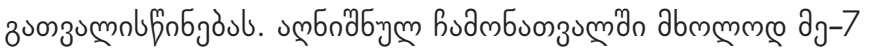

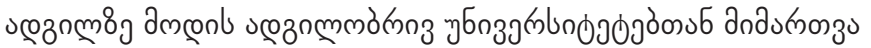

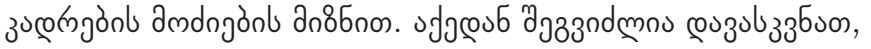

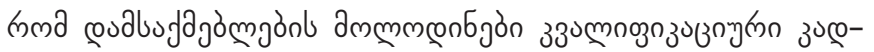

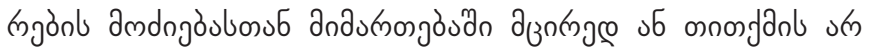

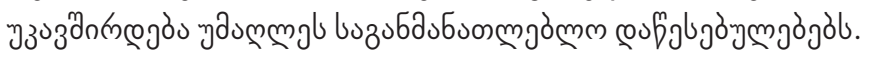
((Ministry of Labor, Health and Social Affairs of Georgia,

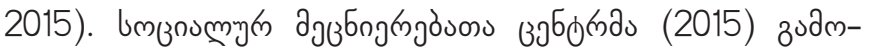

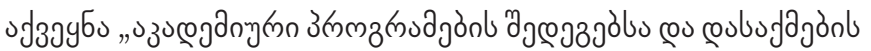

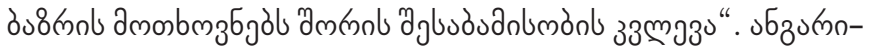

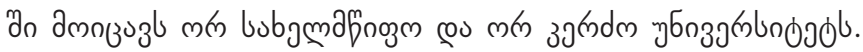

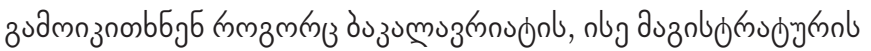

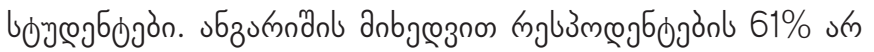

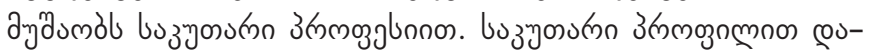

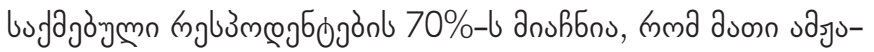

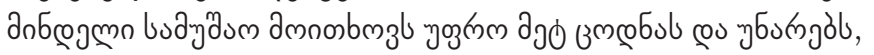

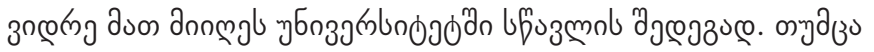

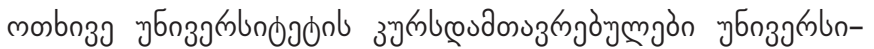

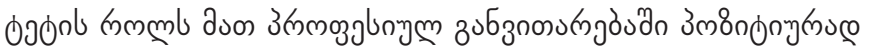
sogulogojg.

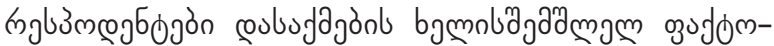

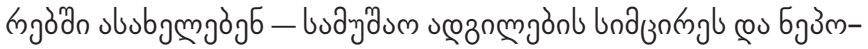

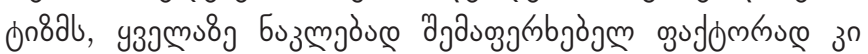

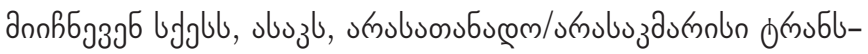

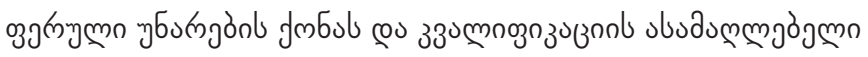
or

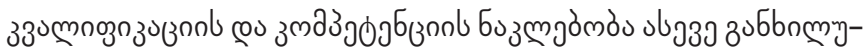

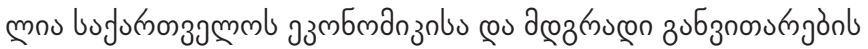

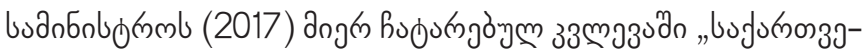

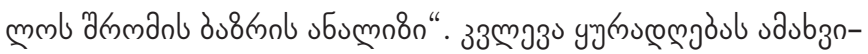

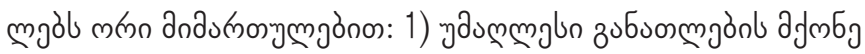
sbumzus

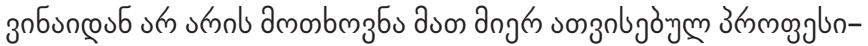

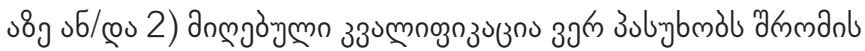

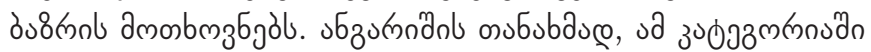

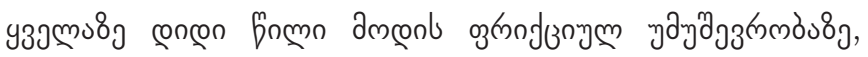

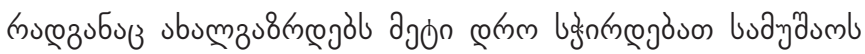
ambudngòmuse.

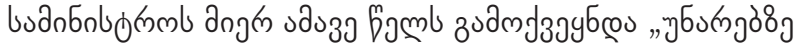

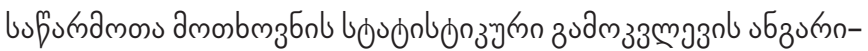

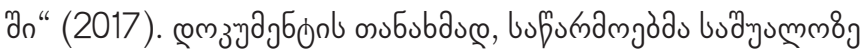

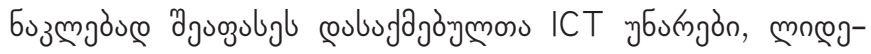

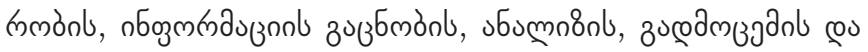

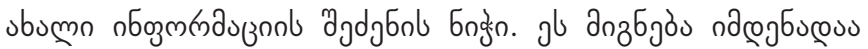

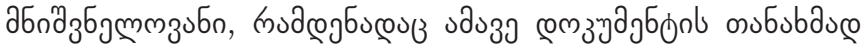

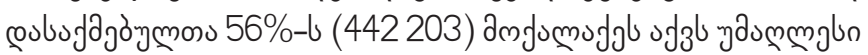
3ง60ongmoso.

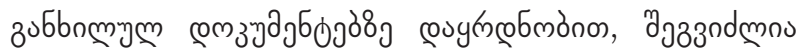

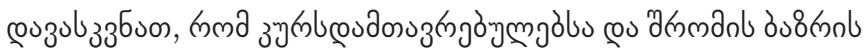

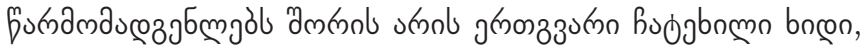

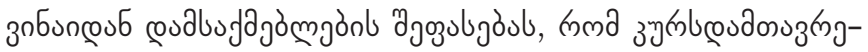

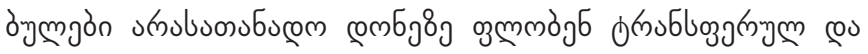

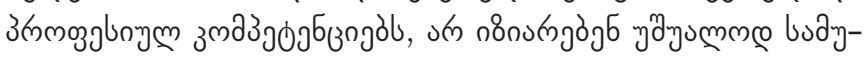

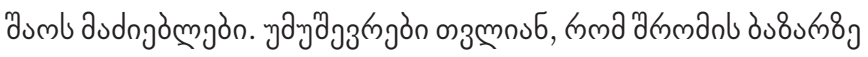

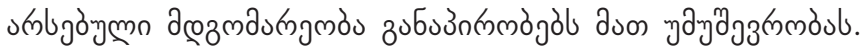

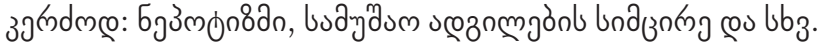

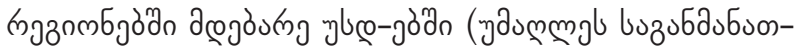

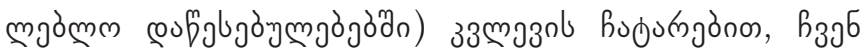

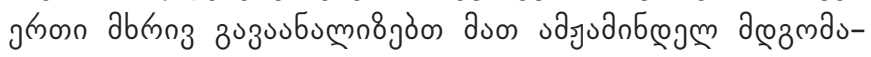

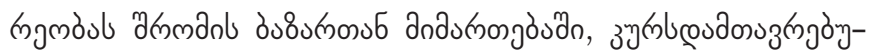

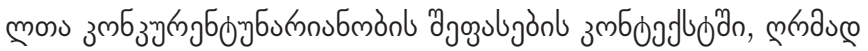

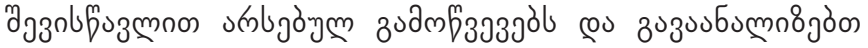

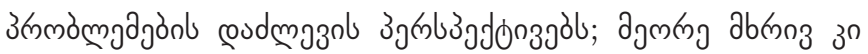

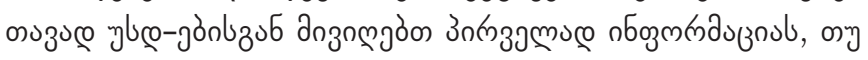

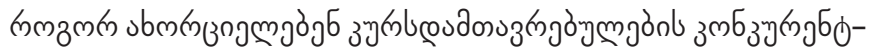

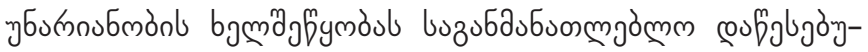

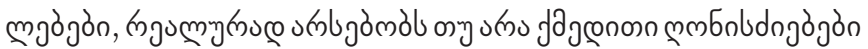

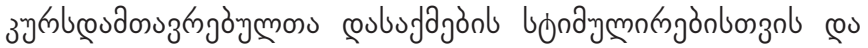
bbzo. 


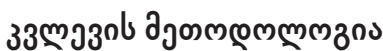

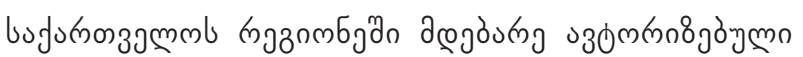

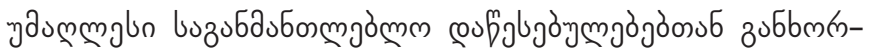

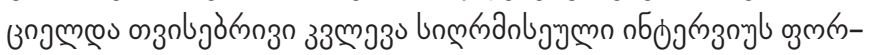

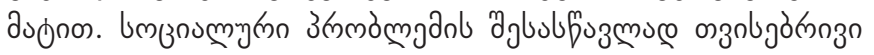

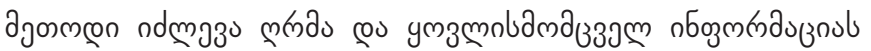

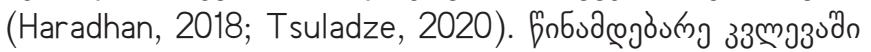

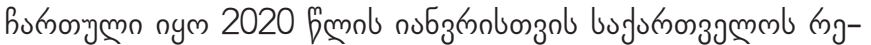

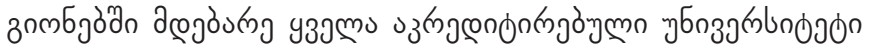

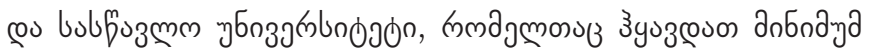

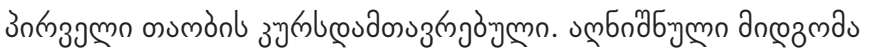

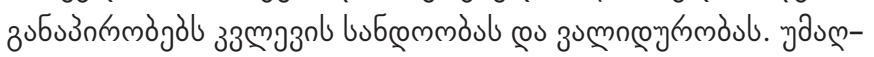

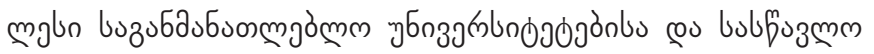

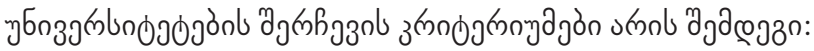

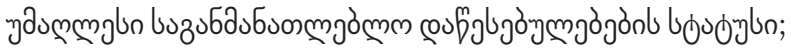

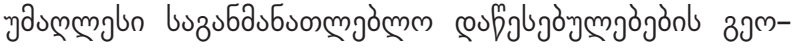

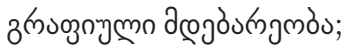

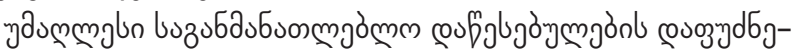
dob ondmann.

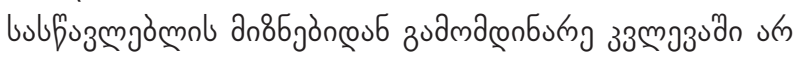

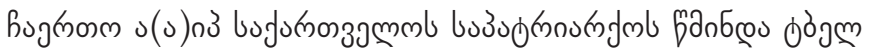

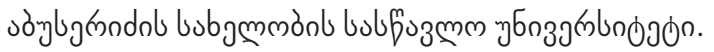

8jam uलб

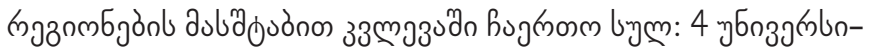

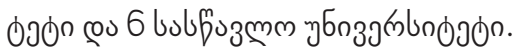

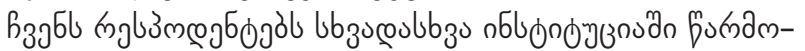

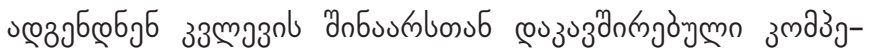

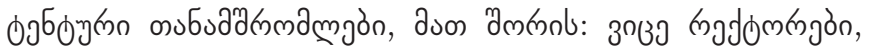

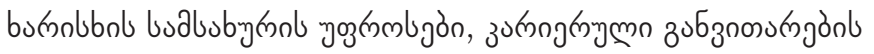

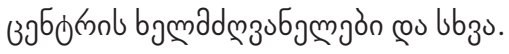

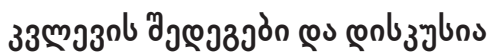

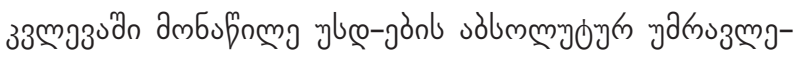

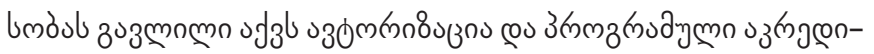

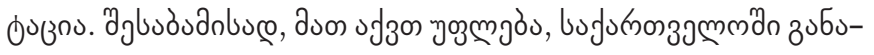

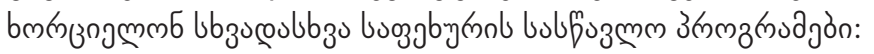

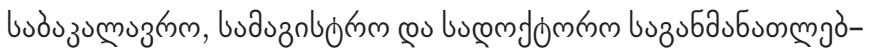

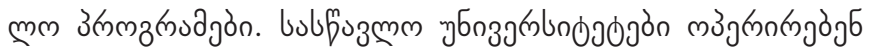

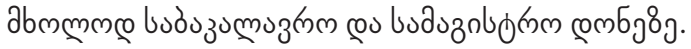

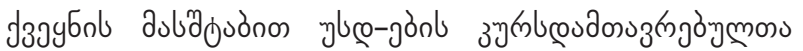

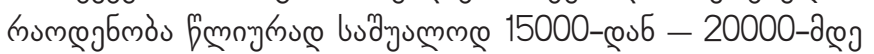

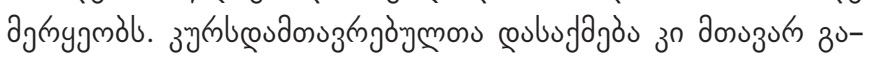

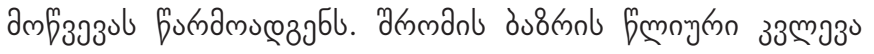

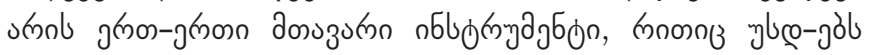

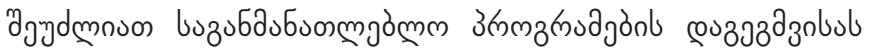

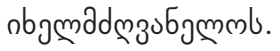

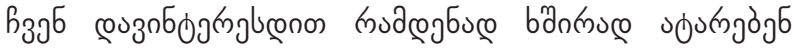

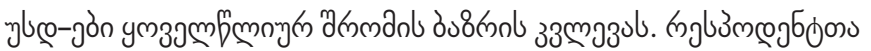

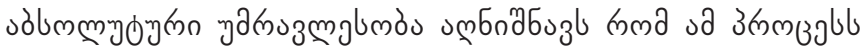

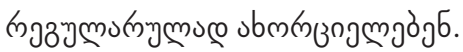

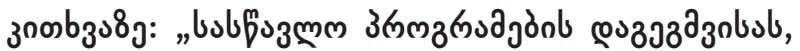

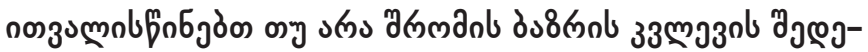

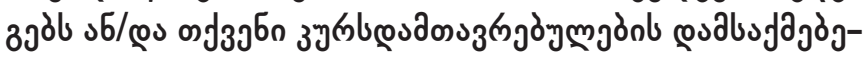

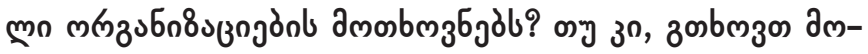

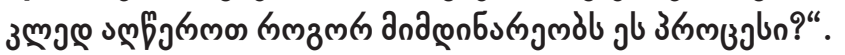

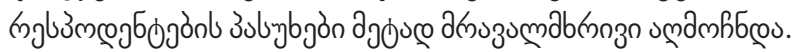

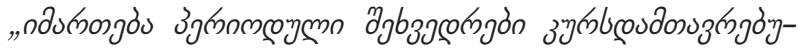

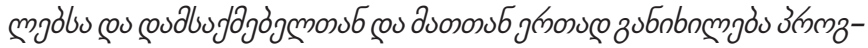

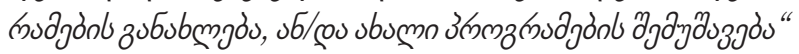

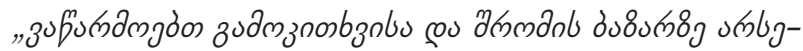

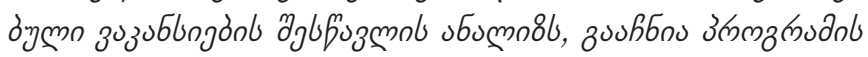
lügrnozozubug"

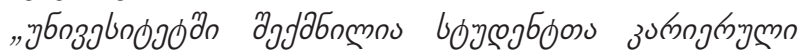

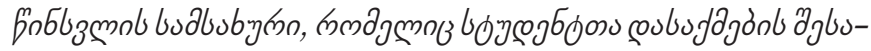

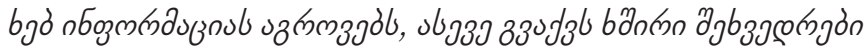

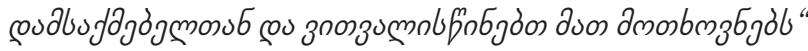

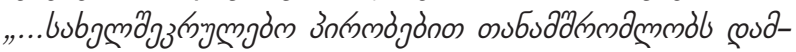

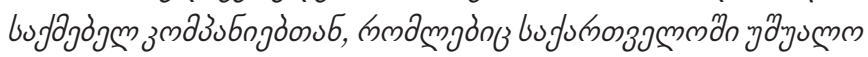

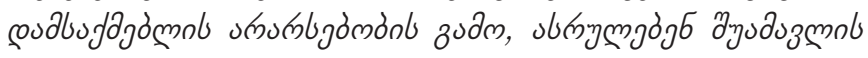

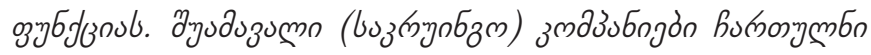

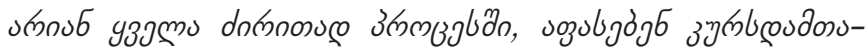

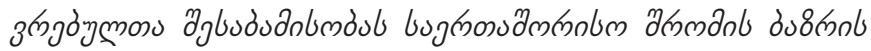

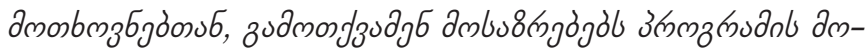

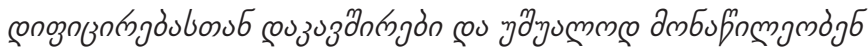

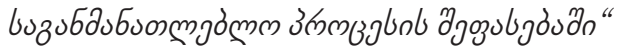

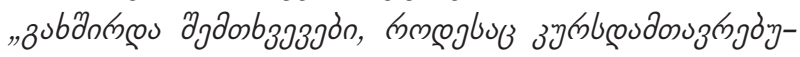

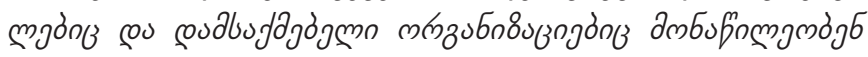

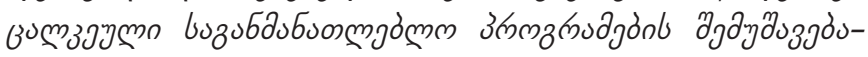
$\eta_{n}$. sanl zurngu, n

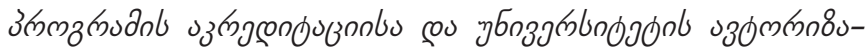

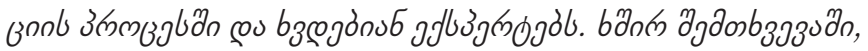

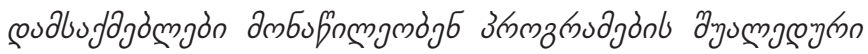

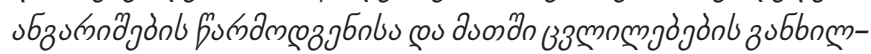

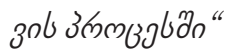

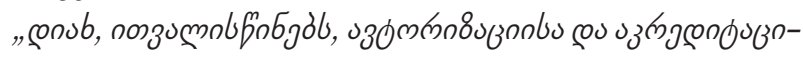
ol boubroundojóng uadb amnosbmalu"

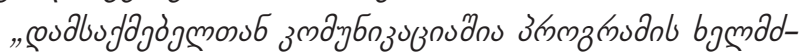

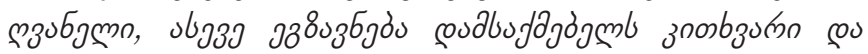

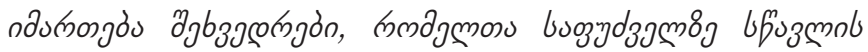
age

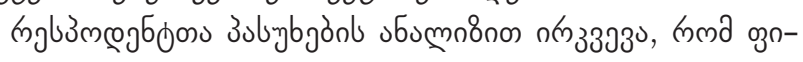

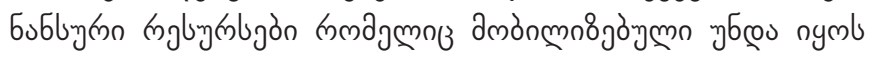

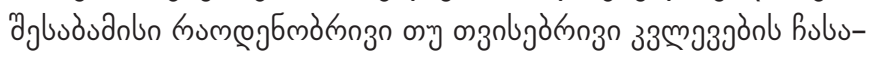

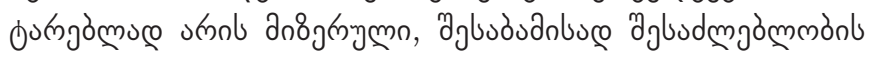

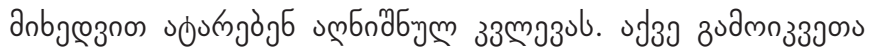

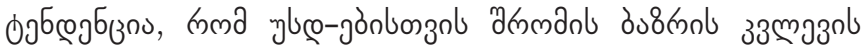

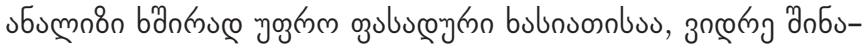

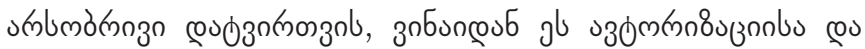




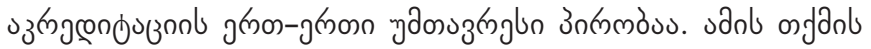

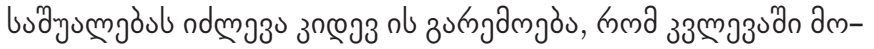

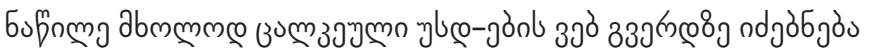

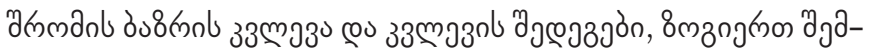

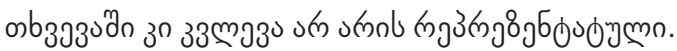

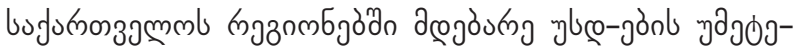

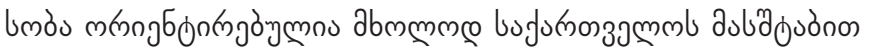

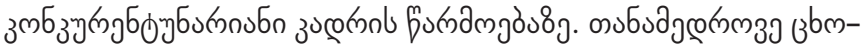
उ

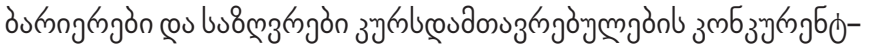

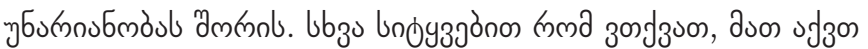
mnonjan

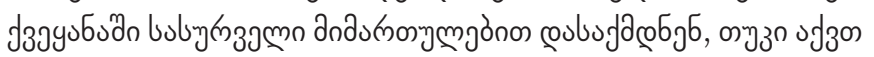

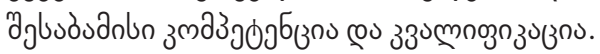

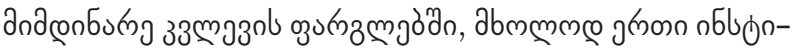

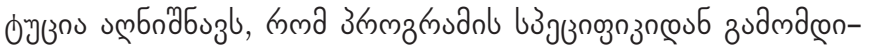

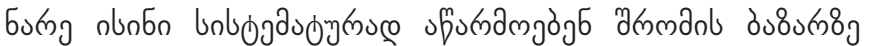
u ml

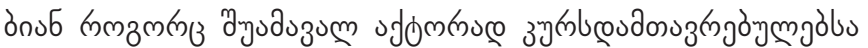

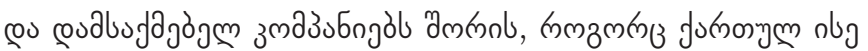

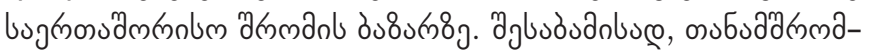

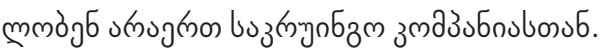

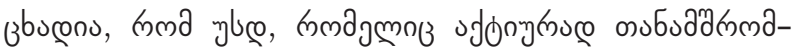

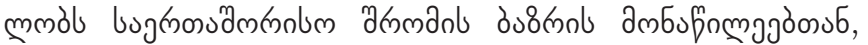

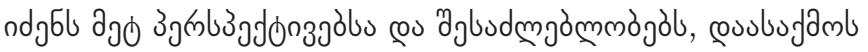
an b

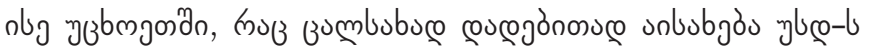

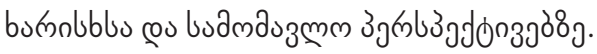

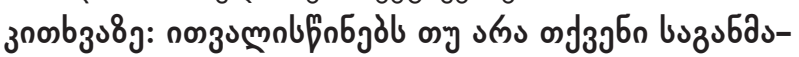

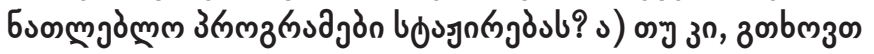

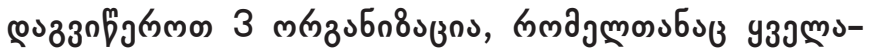

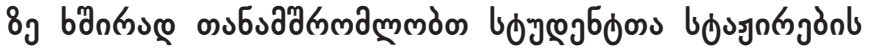

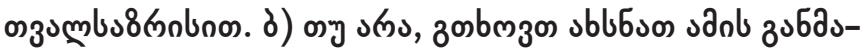

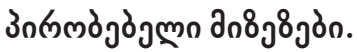

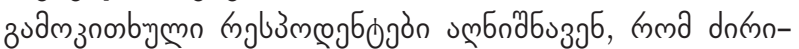

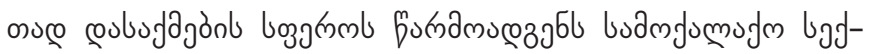

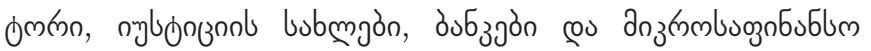

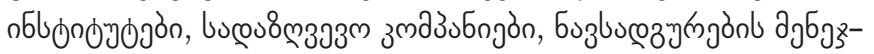

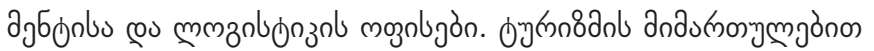

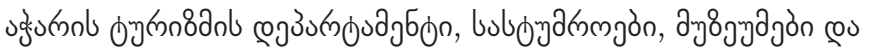
ubzo.

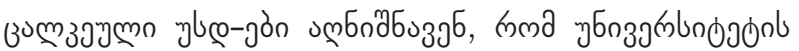

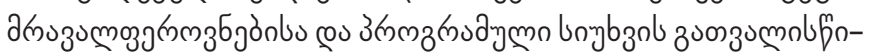

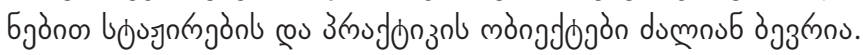

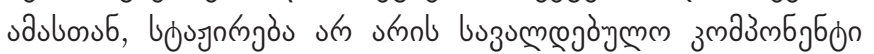

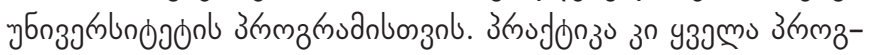
modnb bufonmos.

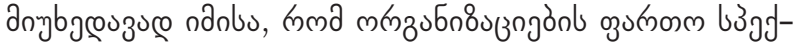

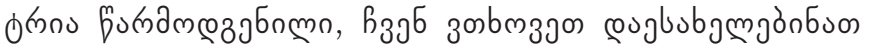

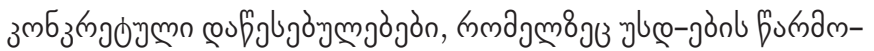

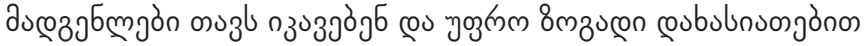

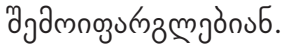

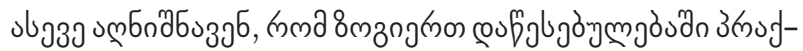

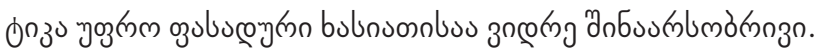

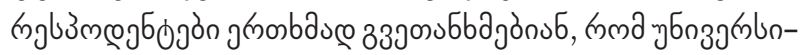

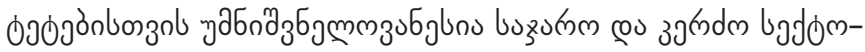

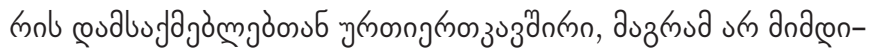

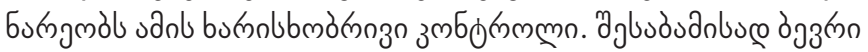

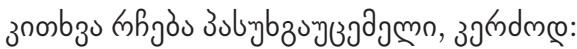

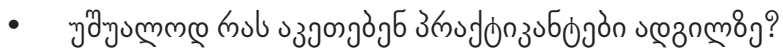

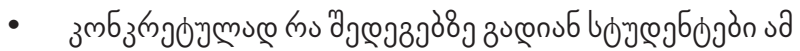

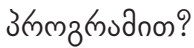

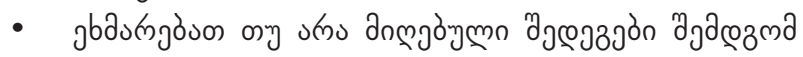

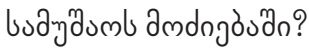

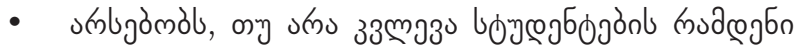

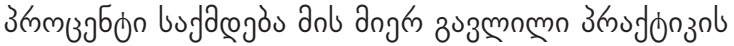

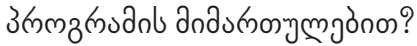

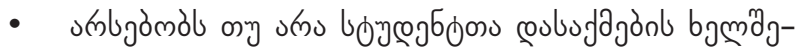

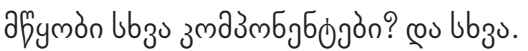

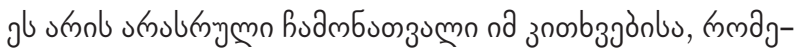

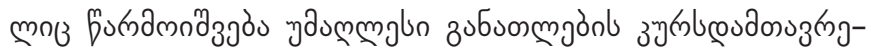

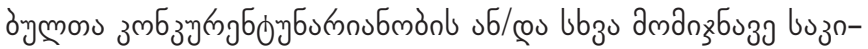
obrol z3mazobul.

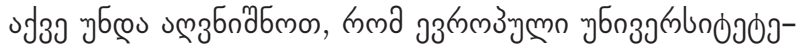

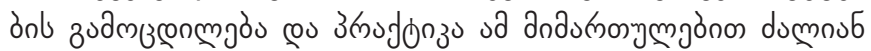

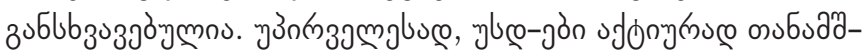

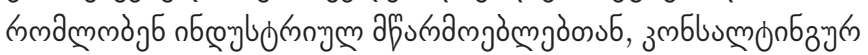

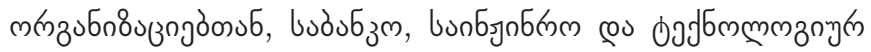

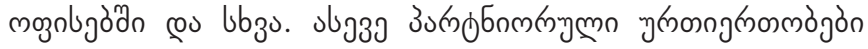

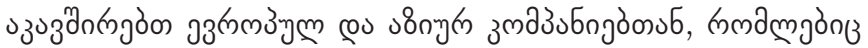

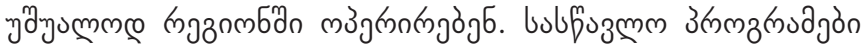

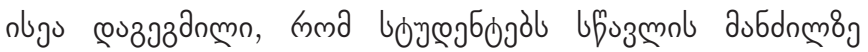
nua

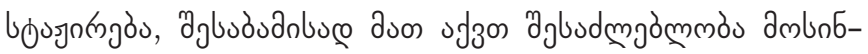

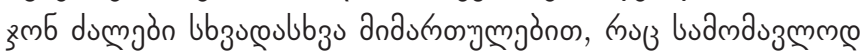

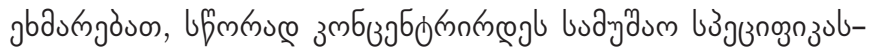

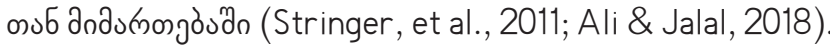

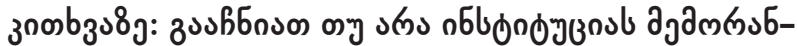

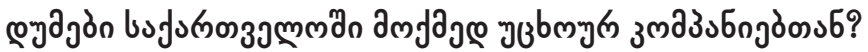

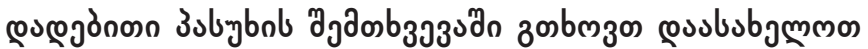

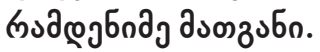

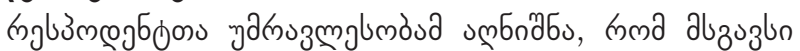

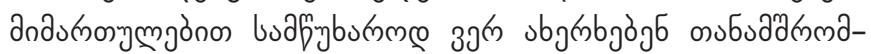

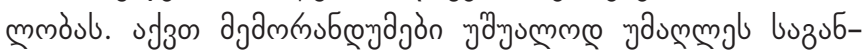

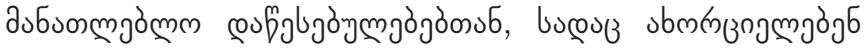

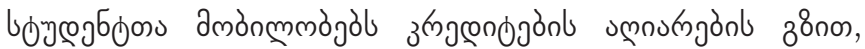

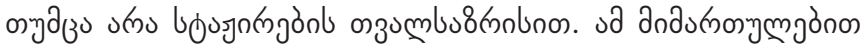

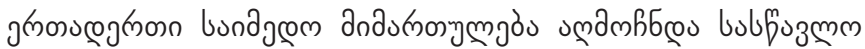




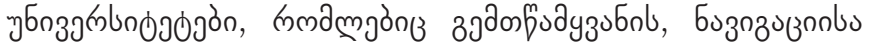

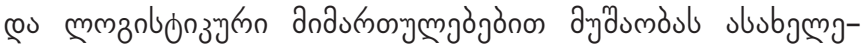

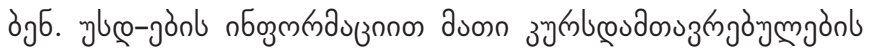

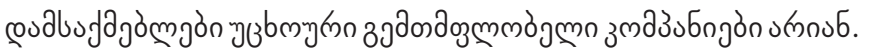

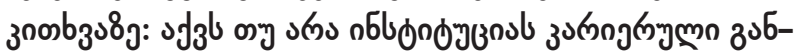

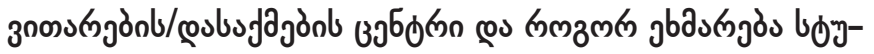

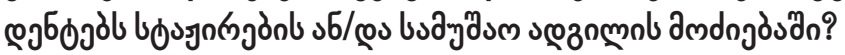

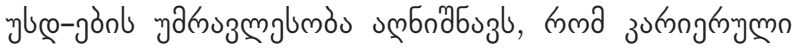

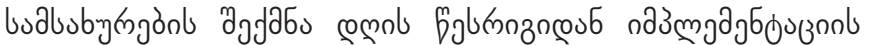

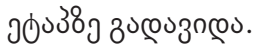

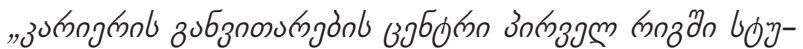

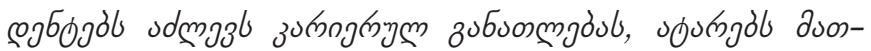

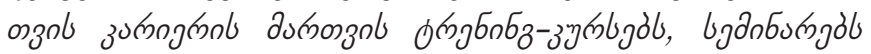

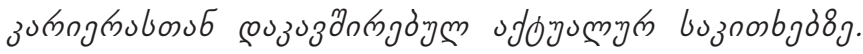

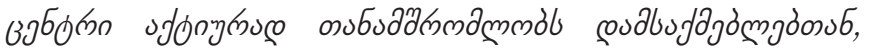

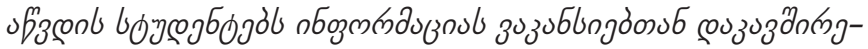

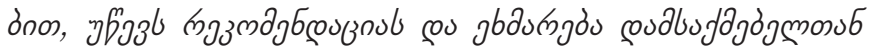

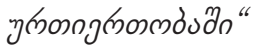

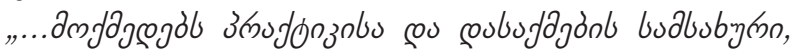

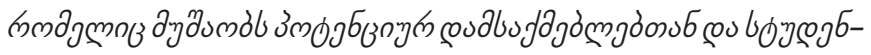

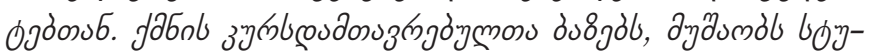

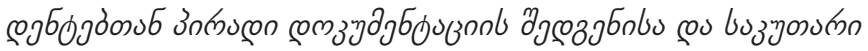
muznl furnূ

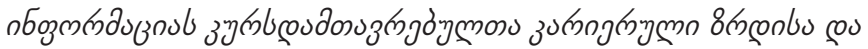

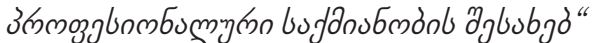

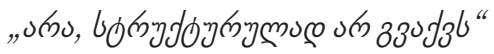

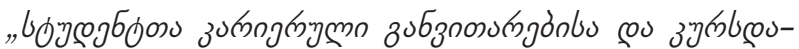

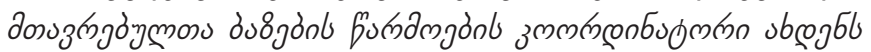
boysegbojönl nбogmman

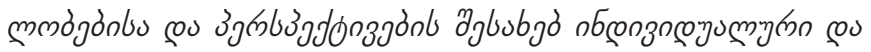

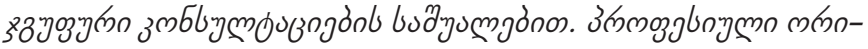

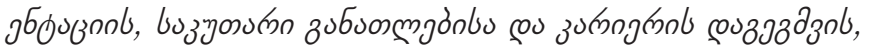

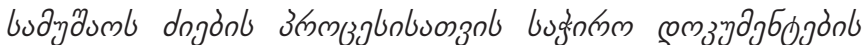

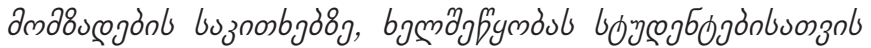

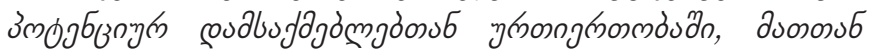

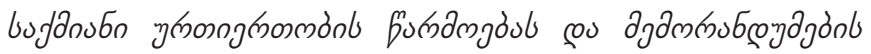

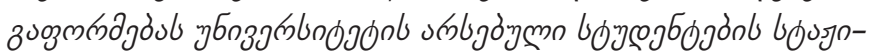

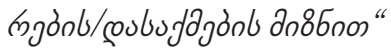

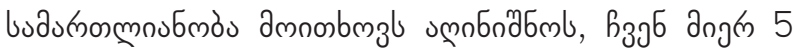

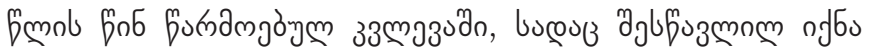

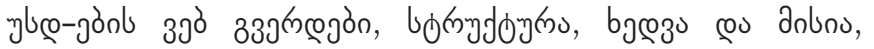

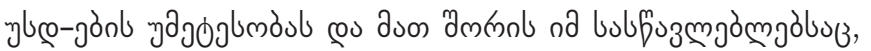

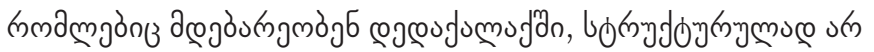
jmbroum zu

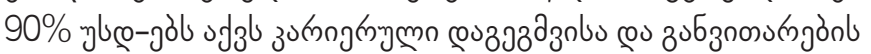

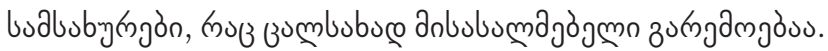

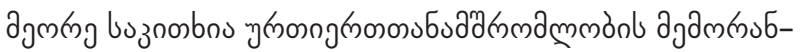

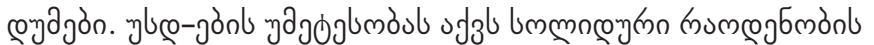

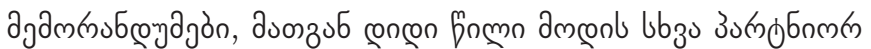

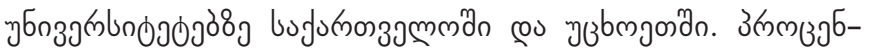

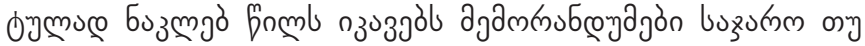

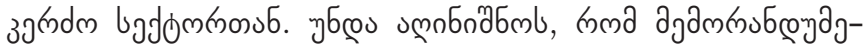

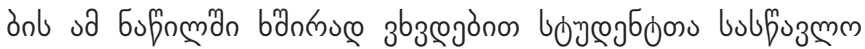

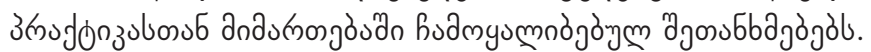

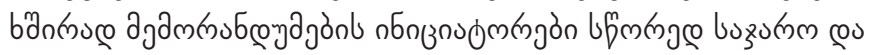

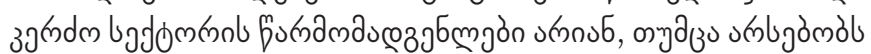

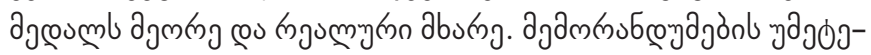

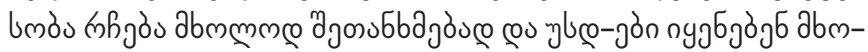

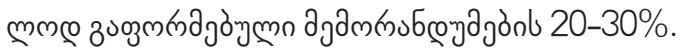

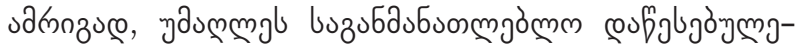

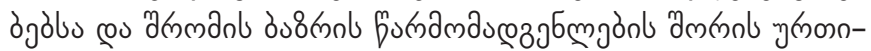

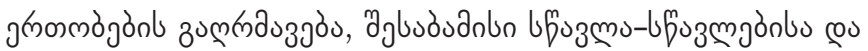

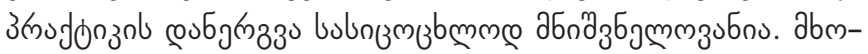

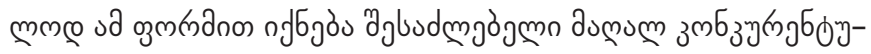

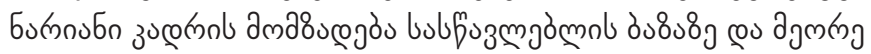

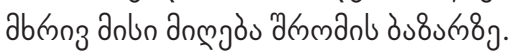

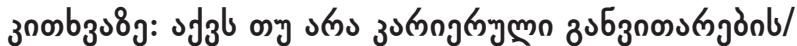

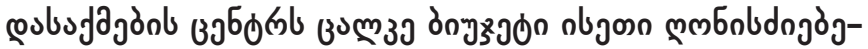

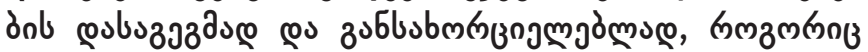

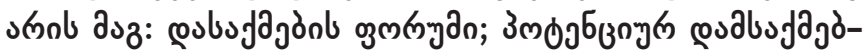

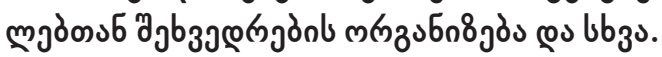

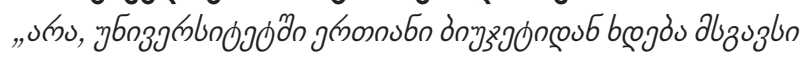

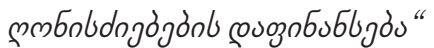

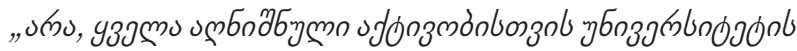

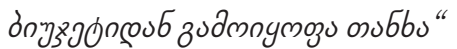

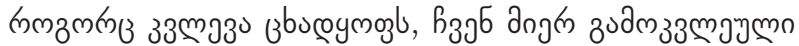

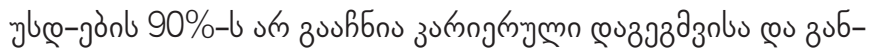

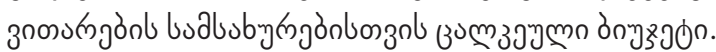

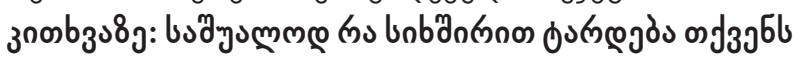

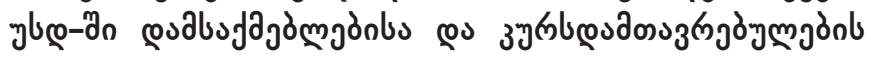

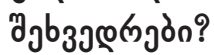

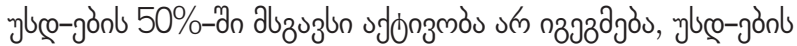

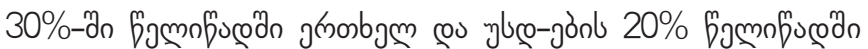

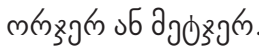

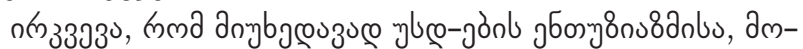

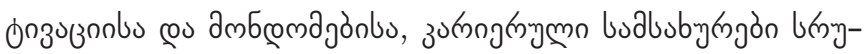

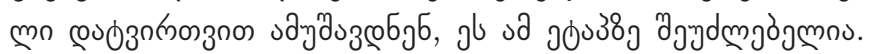

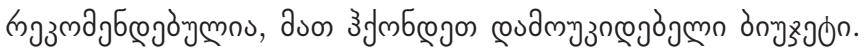

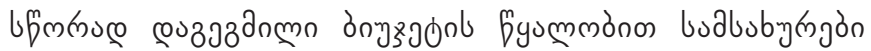

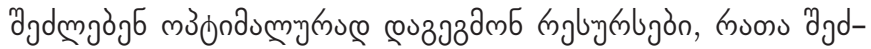

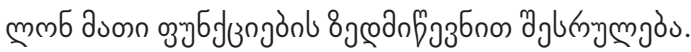

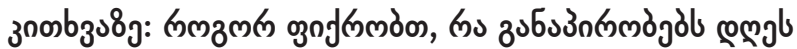
चаuलm

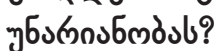

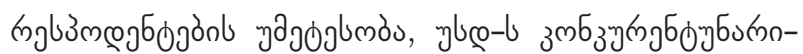

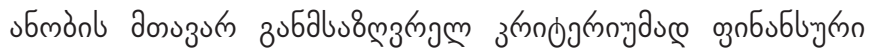

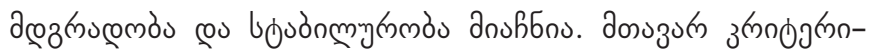

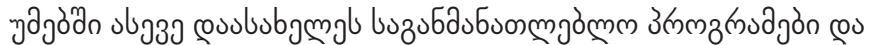

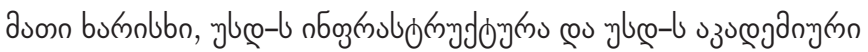
उgrmmbumn. 


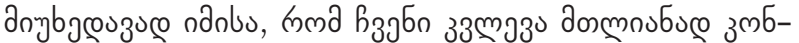

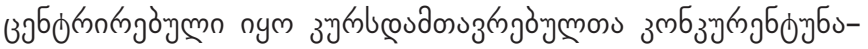

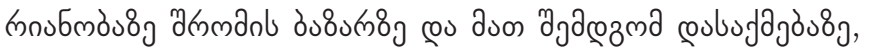

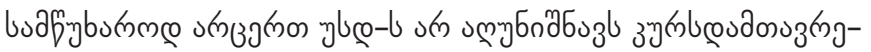

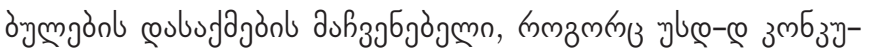

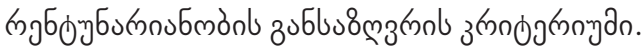

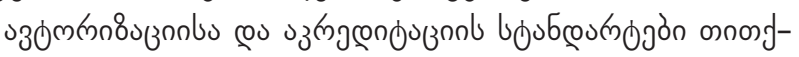

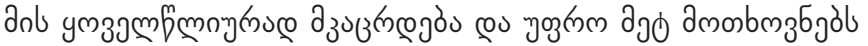

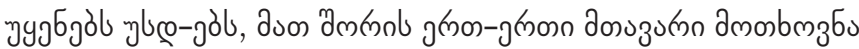

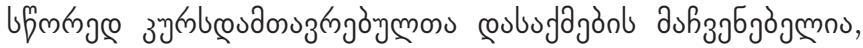

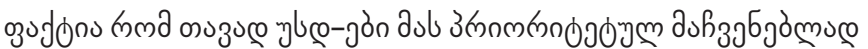

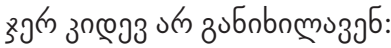

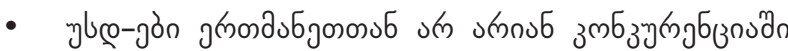

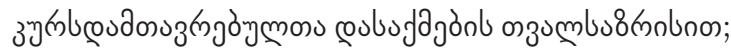

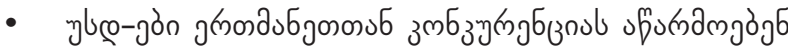

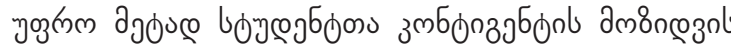

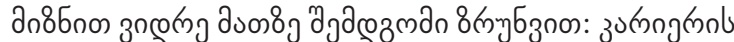

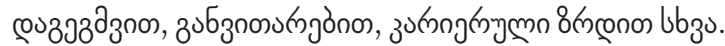

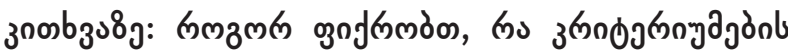

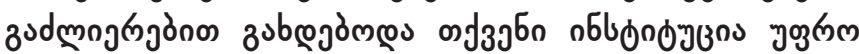
उलб

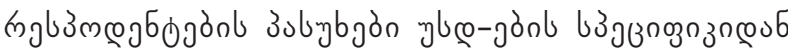

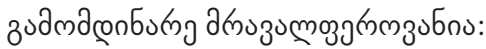

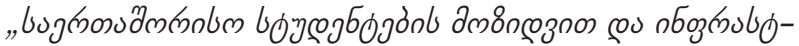

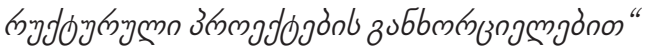

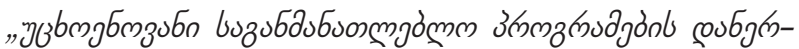

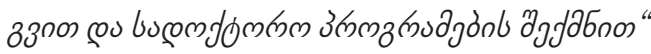

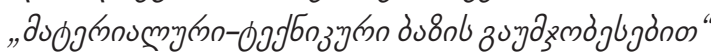

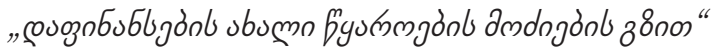

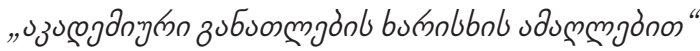

"उзलm

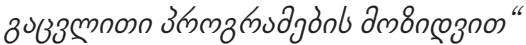

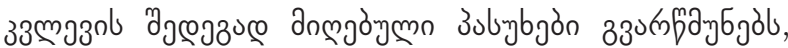

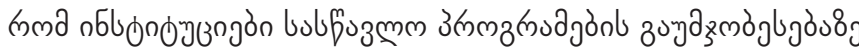

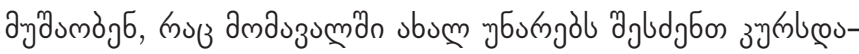

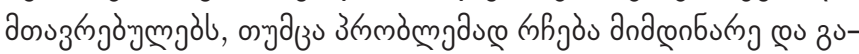

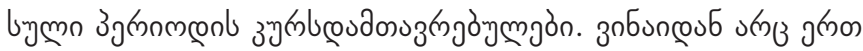
च

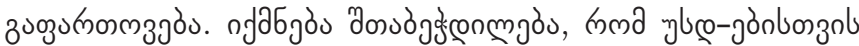

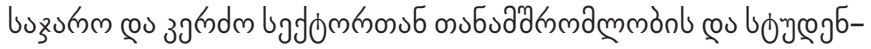

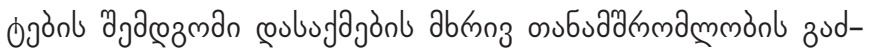

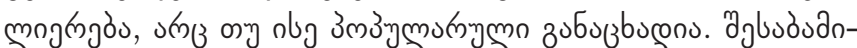

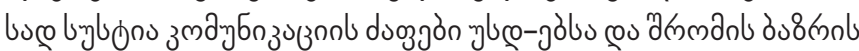

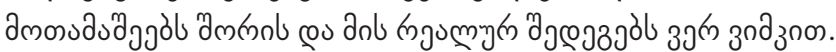

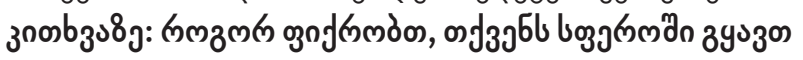

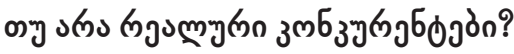

๓ु

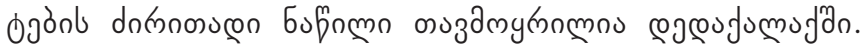

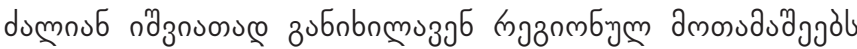

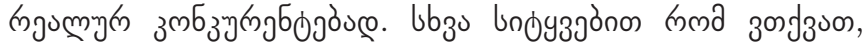

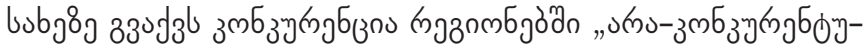

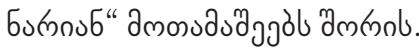

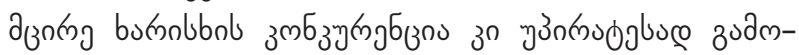

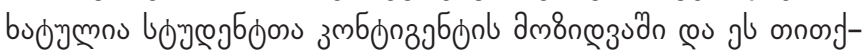

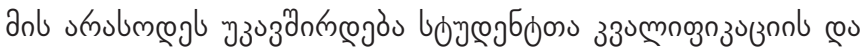

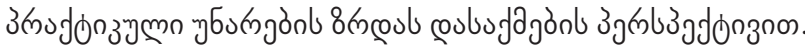

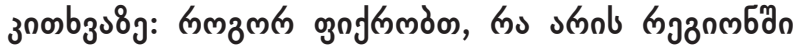

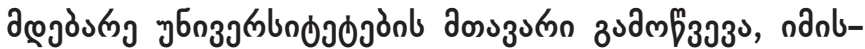

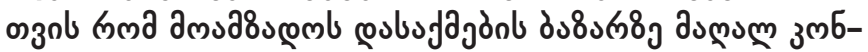

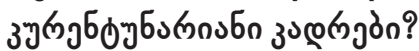

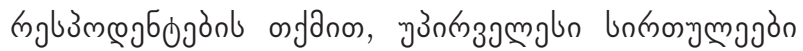

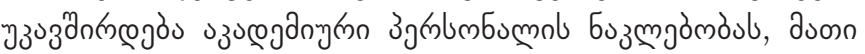

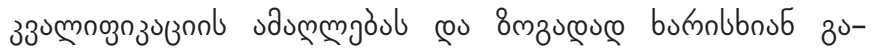

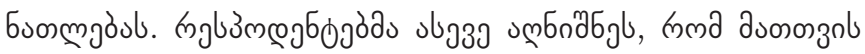

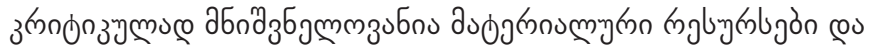
agbuósanlo posozn bubligós.

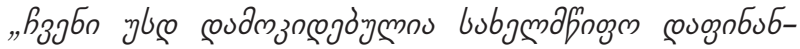

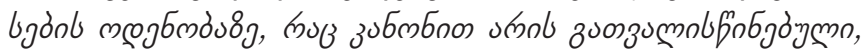

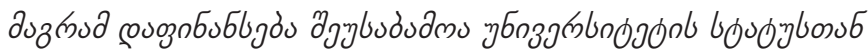

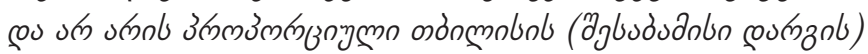

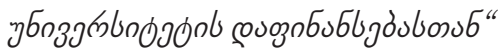

yb

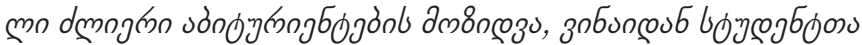
concon buzugon cogcoufumujtan zugcon

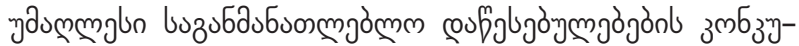

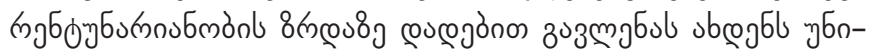

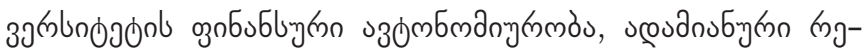

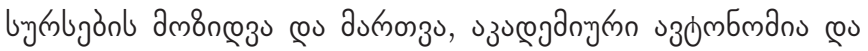

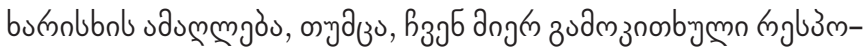

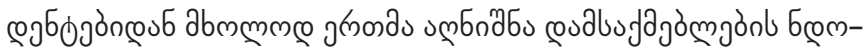

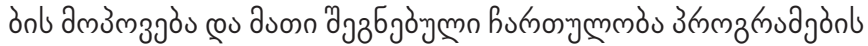

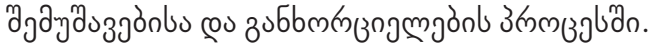

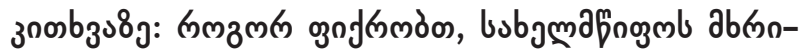

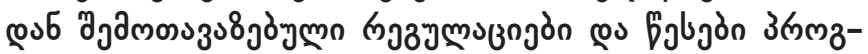

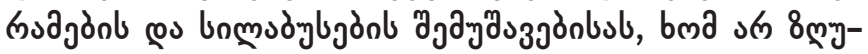

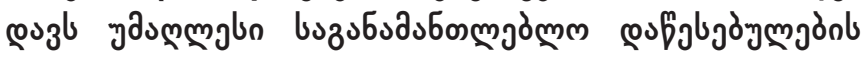

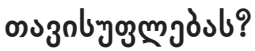

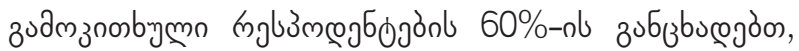

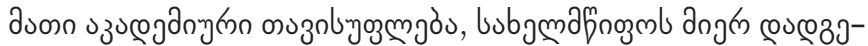

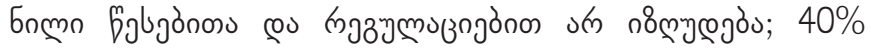

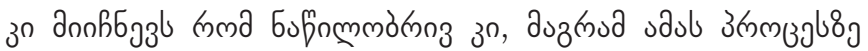

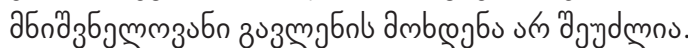

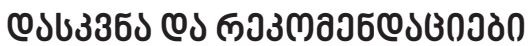

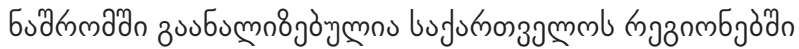

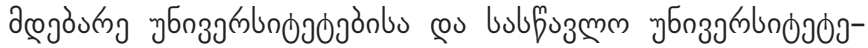

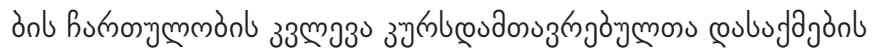




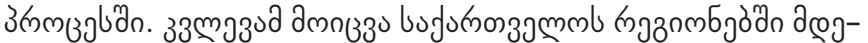

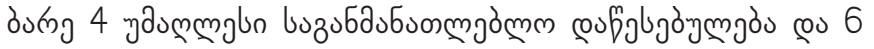

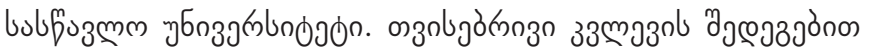

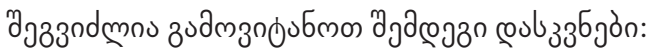

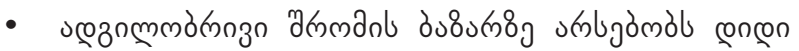

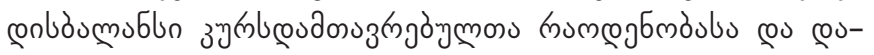

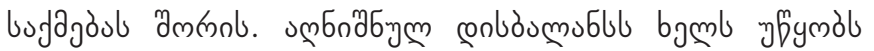

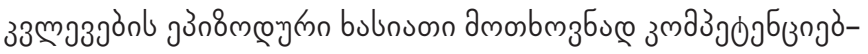

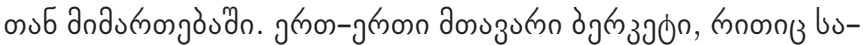

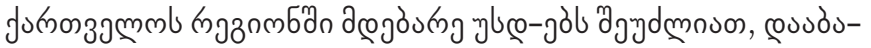

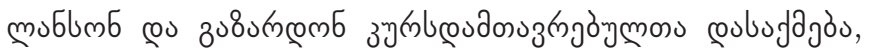

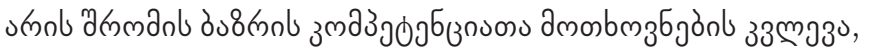

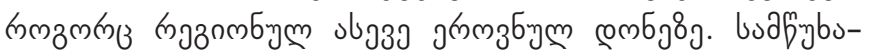

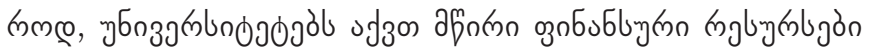

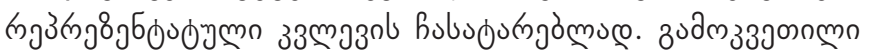

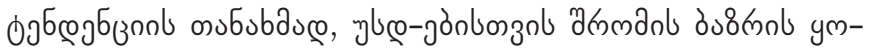

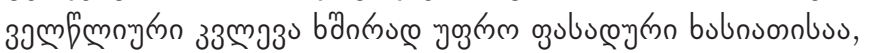

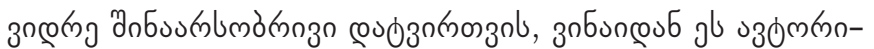

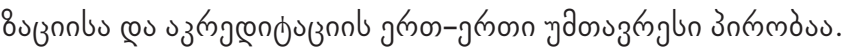

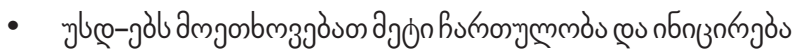

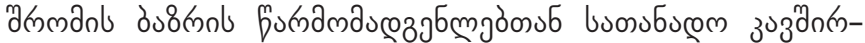

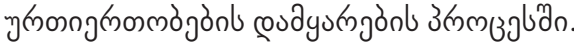

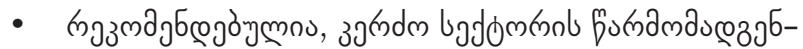

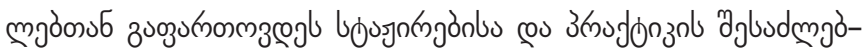

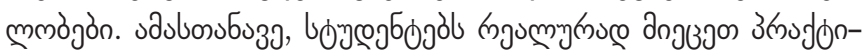

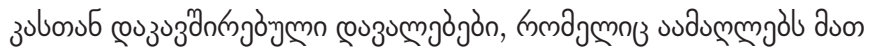

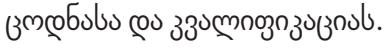

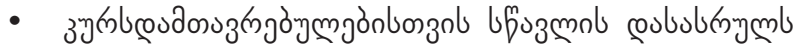

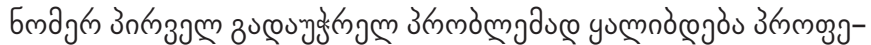

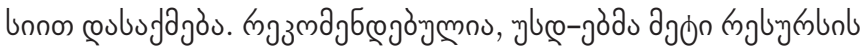

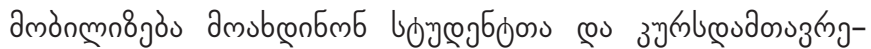

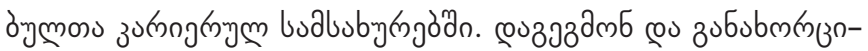

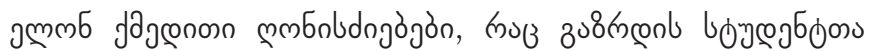

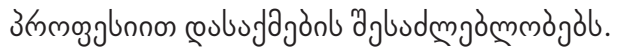

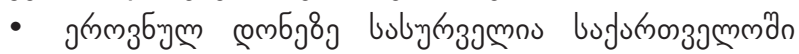

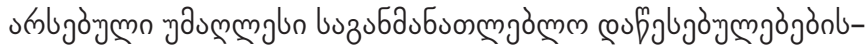

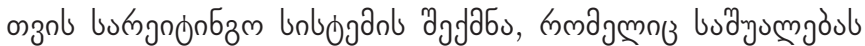

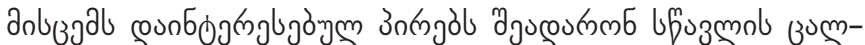

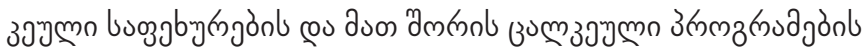

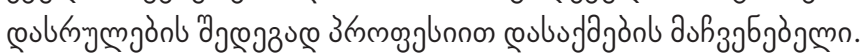

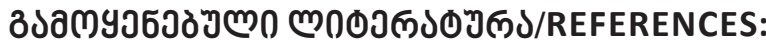

Ali, M., \&Jalal, H. (2018). Higher Education as a Predictor of Employment: The World of Work Perspective. Bulletin of Education and Research, 40(2), 79-90.

Amashukeli, M, \& Lezhava, D. (2015). Correlation study between the results of academic programs and employment market requirements in the social sciences. Tbilisi: Centre for Social Sciences (In Georgian).

Amashukeli, M., Lezhava, D., \& Gugushvili, N. (2017). Education Outcomes, Employment Market and Labor Satisfaction in Georgia. Tbilisi: Open Society - Georgia (In Georgian).

Blagg, K., \& Blom, E. (2018). Evaluating the return on Investment in Higher Education. Wadhington, DC: Urban Institute.

Bregvadze, T. (2013). Higher Education and Employment. Strategic Development of Higher Education and Science. Analysis of Higher Education Policy in Georgia according to Five Strategic Directions. Tbilisi: International Institute for Education Policy Planning and Management (In Georgian).

Center for Social Sciences, (2015). Correlation Study between Academic Program Outcomes and Employment Market Requirements. Tbilisi. (In Georgian).

Hailemariam, A. (2018). Returns to Higher Education in the Very Long-run 1870-2010. Journal of Education and Work, 31:3, 291-306.

Haradhan, M. (2018). Qualitative Research Methodology in Social Sciences and Related Subjects. Journal of Economic Development, Environment and People, 7(01) 23-48.

Kakulia, M., Kapamadze, N, Lomjaria, N., \& Kurkhuli, L. (2016). Unemployment Structure and Structural Unemployment in Georgia. Tbilisi: Georgian Foundation for Strategy and International Studies (In Georgian).

Mincer, J. (1074). Schooling, Experience and Earnings. Human Behavior \& Social Institutions (2).

Ministry of Economy and Sustainable Development of Georgia, (2017). Georgian Labor Market Analysis. Tbilisi. (In Georgian).

Ministry of Economy and Sustainable Development of Georgia, (2017). Statistical Survey of Enterprise Demand for Skills (Report). Tbilisi. (In Georgian).

Stringer, K., Kerpelman, J., \& Storikov, V. (2011). Career preparation: A Longitudinal, Process-Oriented Examination. Journal of Vocational Behavior, 79(1) 158-169.

Tsuladze, L. (2020). Qualitative Methods of Sociological Research. Tbilisi: Ivane Javakhishvili Tbilisi State University Publishing House. (In Georgian). 